\title{
DESIGN PARAMETERS FOR EPS GEOFOAM ${ }^{\mathrm{i})}$
}

\author{
Closure by D. NEGUSSEY ${ }^{\text {ii) }}$
}

The author thanks the discussers for their interest in the paper and contribution of further confirming experimental results. Previous work by the author and his co-workers cited in the paper is also in good agreement with the results presented by the discussers. Sample size and end effects, confining stress state and strain level dependence of EPS geofoam modulus, ambiguity about the range of appropriate Poisson's ratio values as well as interface friction and adhesion performance have been areas of research interest that are now resulting in converging findings. The basis of understanding provided in the paper and the complementary results from the work of the author and the writers will hopefully help to encourage revision of current standards. The approximate doubling of characteristic modulus values should be viewed together with the relatively unchanged apparent strength as shown in Fig. 9 of the paper. The range over which the average stress-strain relationship can be considered to be reasonably linear elastic is much lower than the commonly assumed threshold of 1 percent corrected strain. Further revision of design parameters towards more rational modulus values will facilitate better agreement with prediction of settlements and field performance observations for EPS geofoam that may otherwise be specified relying on current standards and guidelines. The improved understanding and supporting results should be especially useful for detailed design and analysis of more complex and innovative applications along the lines mentioned by the discussers.

i) Vol. 47, No. 1, February 2007, pp.161-170 (Previous discussion by G. A. Athanasopoulos and V. C. Xenaki, Vol. 48, No. 6, December 2008, pp. 859-861)

ii) Geofoam Research Center Director and Professor of Civil and Environmental Engineering Syracuse University, NY, USA (negussey@ syr.edu). 